\title{
NEW MODELS FOR WOLF-RAYET STARS:
}

\section{GLOBAL DIFFUSION}

\author{
L. DENG ${ }^{1}$, A. BRESSAN ${ }^{2}$ and C. CHIOSI ${ }^{3}$ \\ ${ }^{1}$ International School for Advanced Studies, Strada Costiera 11, I-34100, Trieste, Italy \\ ${ }^{2}$ Astronomical Observatory, Vicolo Osservatorio 5, I-35100 Padova, Italy \\ ${ }^{3}$ Department of Astronomy, University of Padova, \\ Vicolo Osservatorio 5, 35122 Padova, Italy
}

\begin{abstract}
We present a scenario for the evolution of massive stars in which a new mixing mechanism (named global diffusion) is taken into account. This type of mixing stands on the critical Reynolds number and radiative viscosity (Schatzman 1977) and allows mixing of material to take place between the core and the surface during the whole evolution on a very slow time scale. The physical processes triggering global diffusion deserve further study. We find that stellar models of massive stars calculated with global diffusion offer interesting clues to understanding the properties of Wolf-Rayet stars and their location in the HRD.
\end{abstract}

Key words: stars: Wolf-Rayet - evolution - mixing processes

Two sets of stellar models computed with the new scheme of mixing are presented. The physical gradients of the models is the same as Bressan et al. (1993), while the mixing mechanism and other details of the models can be found in Deng (1994) and Deng et al. (1994). The stellar tracks are given in Fig. 1, which shows a very good fit to the observation of Hamann et al.

\section{TABLE I}

Theoretical lifetime ratios for the WR sequences

\begin{tabular}{ccccccc}
\hline$Z$ & $M / \mathrm{M}_{\odot}$ & $t_{H}$ & $t_{H e} / t_{H}$ & $\mathrm{WR} / \mathrm{OB}$ & $\mathrm{WNE} / \mathrm{WNL}$ & $\mathrm{WN} / \mathrm{WR}$ \\
\hline 0.020 & 9 & 1.77 & 0.026 & 0.028 & 3.208 & 0.337 \\
& 12 & 1.21 & 0.032 & 0.034 & 1.905 & 0.282 \\
& 15 & 1.02 & 0.041 & 0.045 & 1.099 & 0.232 \\
& 20 & 0.86 & 0.054 & 0.061 & 0.475 & 0.194 \\
& 25 & 0.78 & 0.067 & 0.082 & 0.186 & 0.176 \\
& 30 & 0.81 & 0.087 & 0.109 & 0.150 & 0.185 \\
& 40 & 0.69 & 0.099 & 0.154 & 0.019 & 0.338 \\
& 12 & 0.85 & 0.022 & 0.026 & 0.841 & 0.509 \\
& 15 & 0.71 & 0.028 & 0.042 & 0.382 & 0.391 \\
& 20 & 0.61 & 0.038 & 0.073 & 0.143 & 0.537 \\
& 25 & 0.57 & 0.047 & 0.073 & 0.023 & 0.408 \\
& 40 & 0.51 & 0.066 & 0.148 & 0.001 & 0.521
\end{tabular}




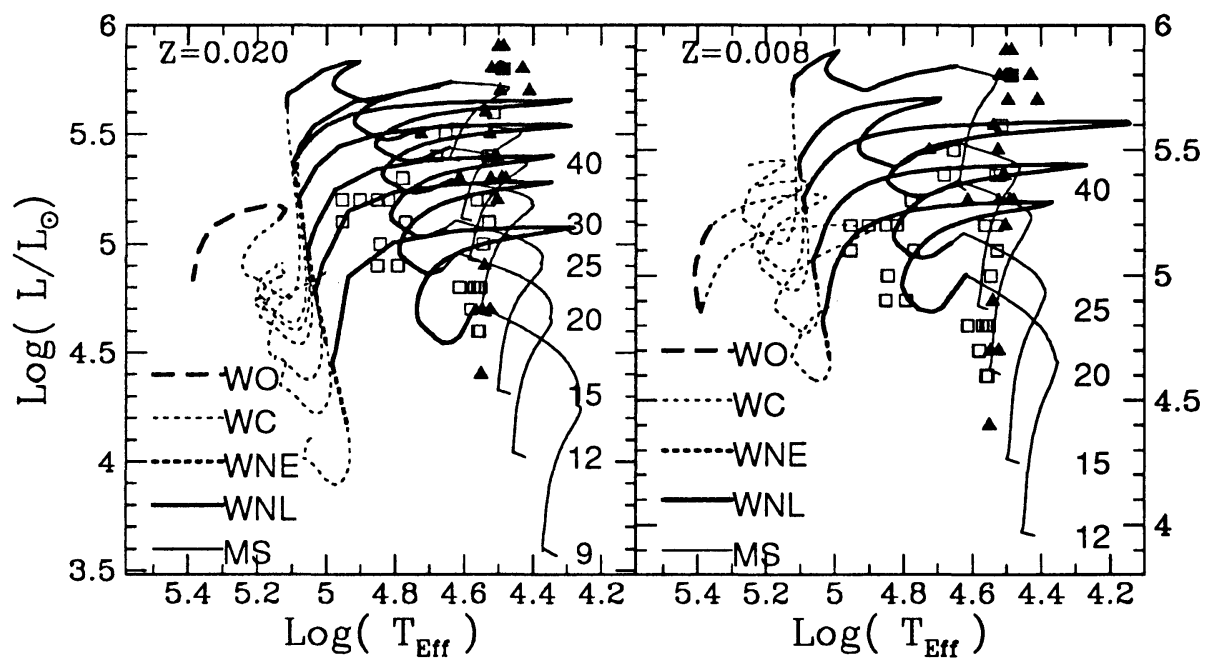

Fig. 1. Evolutionary tracks with global diffusion, superposed on data of Hamann et al. (1993) for single galactic WN stars. Solid triangles show WN stars with surface hydrogen; open squares show hydrogen-free objects.

(1993), especially the low luminosity WNLs, which cannot be explained with the standard models according to Maeder (these proceedings). The life-time data for the sequences are given in Table I.

We find the following properties of WR stars: (1) The formation of WR stars is possible at much lower values of the initial mass (therefore much lower luminosity) than with standard models (cf. Schaller et al. 1992). The initial mass above which the WR phase starts is $\sim 10 \mathrm{M}_{\odot}$, this eventually alleviates the discrepancy between the expected and the observed positions of WR stars in the HRD, in particular for the less luminous ones. No ad hoc assumptions for the rates of mass loss are needed. (2) However, global diffusion does not bear very much on the evolution of the most massive stars, say above 30-40 $\mathrm{M}_{\odot}$, as it is overwhelmed by the dominant effect of mass loss. (3) Finally, there is a lower limit below which global diffusion does not alter the classical evolutionary path in the HRD and therefore does not lead to the formation of a WR object.

\section{References}

Bressan, A., Fagotto, F., Bertelli, G., Chiosi, C. 1994, A\&A Suppl. 100, 647

Deng, L. 1994, PhD thesis

Deng, L., Bressan, A., Chiosi, C. 1994 , in preparation

Hamann, W.R., Koesterke, L., Wessolowski, U. 1993, $A \& A$ 274, 397

Schaller, G., Schaerer, D., Meynet, G., Maeder, A. 1992, A\&A Suppl. 96, 269

Schatzman, E. 1977, $A \mathscr{B} A$ 56, 211 